\title{
A Unified Transform for LTI Systems-Presented as a (Generalized) Frame
}

\author{
Arie Feuer, ${ }^{1}$ Paul M. J. Van den Hof, ${ }^{2}$ and Peter S. C. Heuberger ${ }^{2}$ \\ ${ }^{1}$ Department of Electrical Engineering, Technion-Israel Institute of Technology, Haifa 32000, Israel \\ ${ }^{2}$ Delft Center for Systems and Control, Delft University of Technology, 2628 Delft, the Netherlands
}

Received 19 August 2004; Revised 30 May 2005; Accepted 31 May 2005

We present a set of functions in $L^{2}([0, \infty))$ and show it to be a (tight) generalized frame (as presented by G. Kaiser (1994)). The analysis side of the frame operation is called the continuous unified transform. We show that some of the well-known transforms (such as Laplace, Laguerre, Kautz, and Hambo) result by creating different sampling patterns in the transform domain (or, equivalently, choosing a number of subsets of the original frame). Some of these resulting sets turn out to be generalized (tight) frames as well. The work reported here enhances the understanding of the interrelationships between the above-mentioned transforms. Furthermore, the impulse response of every stable finite-dimensional LTI system has a finite representation using the frame we introduce here, with obvious benefits in identification problems.

Copyright ( 2006 Hindawi Publishing Corporation. All rights reserved.

\section{INTRODUCTION}

Linear time invariant (LTI) systems representations have been subject of research for many years resulting in a vast amount of literature. Tools such as frequency response (FR), Laplace transform (LP), Laguerre bases, and Hambo bases (see, e.g., [2] and references therein) play key roles in these investigations. In (almost) parallel the signal processing community has been developing tools for signal representation such as Fourier transform (FT), short-time FT (STFT), continuous wavelet transform (CWT), and frames (see, e.g., [3$5])$. We present here an initial effort, on our part, to find common grounds by using ideas and concepts from the latter to generate a unified transform (UT) aimed mainly at system representations.

The use of orthonormal bases for signal and system representations has obvious benefits and a number of such bases have been presented and discussed in the literature. However, especially in the signal processing community, it has been recognized for some time, that using larger sets of functions may have a number of benefits. These sets are referred to as frames (see, e.g., [4-8]). Typically, frames discussed in the literature are countable sets. However, in [1,9], more general frames, coined as continuous frames or generalized frames, are presented. Since we will use extensively the structure, concepts, and terminology associated with these generalized frames and since we anticipate the reader to be less familiar with these type of frames, we feel that a brief review would be useful. For a more detailed discussion we refer the reader to $[1]$.

Let us start with a definition.

Definition 1 ([1, Definition 4.1]). Let $\mathscr{H}$ be a Hilbert space and let $M$ be a measure space with measure $\mu$. A generalized frame in $\mathscr{H}$ indexed (or "labeled") by $M$ is a family of vectors (functions in $\mathscr{H}$ ) $\mathscr{H}_{M} \equiv\left\{h_{m} \in \mathscr{H}: m \in M\right\}$ such that

(1) for every $f \in \mathscr{H}$, the function $\tilde{f}: M \rightarrow \mathbb{C}$ defined by

$$
\tilde{f}(m) \equiv\left\langle h_{m}, f\right\rangle_{\mathscr{H}}
$$

is measurable,

(2) there is a pair of constants $0<A \leq B<\infty$ such that for every $f \in \mathcal{H}$,

$$
A\|f\|_{\mathscr{H}}^{2} \leq\|\tilde{f}\|_{L^{2}(\mu)}^{2} \leq B\|f\|_{\mathscr{H}}^{2} .
$$

Note that the STFT and the continuous wavelet transform (CWT) are two examples of generalized frames.

In STFT

$$
\begin{aligned}
& \mathcal{H}=L^{2}(\mathbb{R}), \quad M=\mathbb{R}^{2}, \\
& m=(\omega, u), \quad h_{m}(t)=g(t-u) e^{j \omega t}, \\
& \mu(A)=\int_{A} d u d \omega,
\end{aligned}
$$




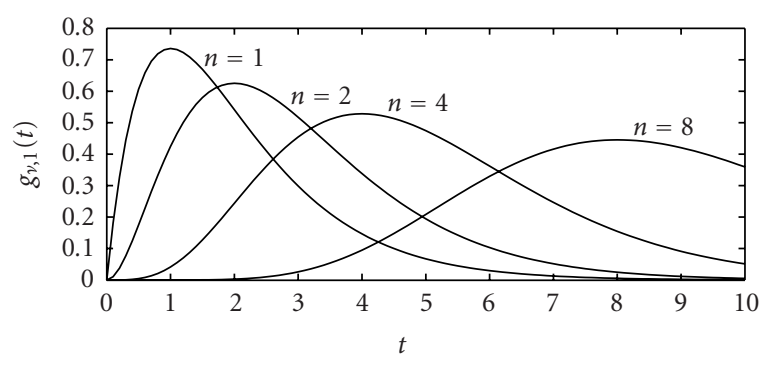

FIGURE 1: Window function $g_{\nu, 1}(t)$ for $\nu=1,2,4,8$.

where $g(t)$ is a predetermined localizing window. In CWT

$$
\begin{aligned}
& \mathcal{H}=L^{2}(\mathbb{R}), \quad M=\mathbb{R}_{+}^{2}, \\
& m=(a, u), \quad h_{m}(t)=\psi\left(\frac{t-u}{a}\right), \\
& \mu(A)=\int_{A} \frac{d a d u}{a^{2}},
\end{aligned}
$$

where $\psi(t)$ is the mother wavelet.

As is well known, (1) represents the analysis side of the process while condition (2) guarantees the existence of the synthesis side. To actually derive the synthesis (reconstruction) expression one needs to find a reciprocal (dual) frame $\mathscr{H}^{M} \equiv\left\{h^{m} \in \mathscr{H}: m \in M\right\}$ for which one has $\int_{M} d \mu h_{m}\left(h^{m}\right)^{*}=I$ (commonly referred to as the resolution of unity). Then, the synthesis is given by

$$
\begin{aligned}
f & =\int_{M} d \mu h^{m} \tilde{f}(m) \\
& =\int_{M} d \mu h^{m}\left\langle h_{m}, f\right\rangle_{\mathscr{H}} \\
& =\int_{M} d \mu h_{m}\left\langle h^{m}, f\right\rangle_{\mathscr{H}} .
\end{aligned}
$$

We note that typically, for frames, $\int_{M} d \mu h^{m} g(m)=f$ does not uniquely determine $g(m) \cdot g(m)=\tilde{f}(m)$ is only one such choice but it turns out to have a special property. Of all possible coefficient functions $g \in L^{2}(\mu)$, for a given $f \in \mathcal{H}$, $\tilde{f}$ has the least "energy" $\|g\|_{L^{2}(\mu)}$ (i.e., it is the least squares choice—see [1]).

\section{THE UNIFIED TRANSFORM}

\subsection{The continuous unified transform}

Since the exponential function plays a central role in LTI systems and their impulse responses, we start with

$$
g(t)=\sqrt{2} e^{-t}, \quad t \geq 0 .
$$

In the sequel we limit our discussion to the space $L^{2}([0, \infty))$ and functions in this space, hence, will drop the explicit statement $t \geq 0$. Using the norm in this space, denoted by $\|\cdot\|_{2}$, note that $\|g\|_{2}=1$. We now use this basic function to generate a family of functions as follows:

$$
g_{v, a}(t)=\sqrt{\frac{2 a}{\Gamma(2 v+1)}}(2 a t)^{v} e^{-a t},
$$

where $v \geq 0, a>0$ are real and $\Gamma(x)$ is the Gamma function defined by

$$
\Gamma(x)=\int_{0}^{\infty} t^{x-1} e^{-t} d t, \quad x>0\left(\Gamma(n+1)=n !, n \in \mathbb{N}_{0}\right) .
$$

Finally, define the functions

$$
\varphi_{v, a, \omega}(t)=g_{v, a}(t) e^{j \omega t}=\sqrt{\frac{2 a}{\Gamma(2 v+1)}}(2 a t)^{\nu} e^{-a t} e^{j \omega t},
$$

where $\omega \in \mathbb{R}$.

We now make the following observations: a set of functions has been defined, "labeled" by the values $(\nu, a, \omega)$. Recalling that the set of functions used for short-time Fourier transforms (STFT) are of the form

$$
\varphi_{\omega, u}(t)=g(t-u) e^{j \omega t},
$$

where $g(t)$ is a predetermined (localizing) window, we note the resemblance of these functions to those defined in (9) - a window function multiplied by the function $e^{j \omega t}$. The "window" function $g_{v, a}(t)$ in (9) can be shifted along the time axis by choices of $(\nu, a)$, as illustrated in Figure 1 for $a=1$, $v=1,2,4,8$.

However, differing from the STFT, we do not have fixedshaped windows and shifts along the time axis are not linear in any of the "labeling variables" $(\nu, a)$. In fact, $\max g_{\nu, a}(t)$ is located at $t=v / a$. On the other hand, we notice that the impulse response of a finite-dimensional stable LTI system is a finite linear combination of functions from this family. Hence, this family is a natural choice to be used for linear system representation (through their impulse responses). This is our main motivation and we will come back to this point in the sequel.

Let us use the set of functions introduced in (9) to define the continuous unified transform (CUT) as follows:

$$
\begin{aligned}
\tilde{f}(\nu, a, \omega) & =\left\langle\varphi_{v, a, \omega}, f\right\rangle=\int_{0}^{\infty} \overline{\varphi_{\nu, a, \omega}(t)} f(t) d t \\
& =\sqrt{\frac{2 a}{\Gamma(2 v+1)}} \int_{0}^{\infty}(2 a t)^{v} e^{-a t} e^{-j \omega t} f(t) d t,
\end{aligned}
$$

$\nu, a, \omega$ as before.

Denote

$$
M=\{(\nu, a, \omega): \nu \geq 0, a>0, \omega \in \mathbb{R}\}
$$




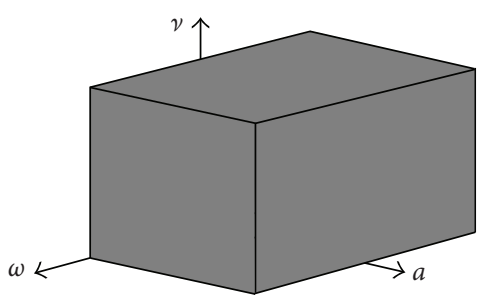

(a)

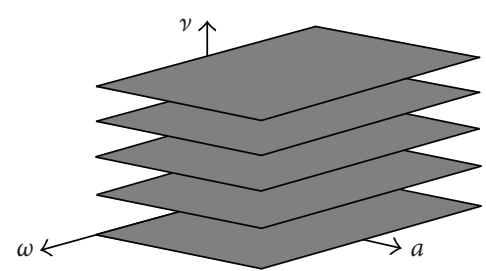

(b)

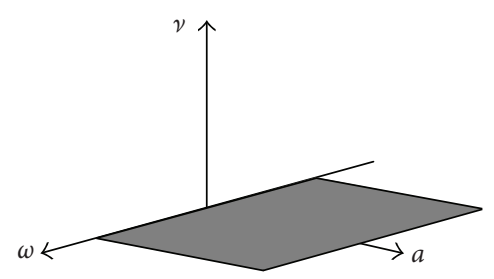

(c)

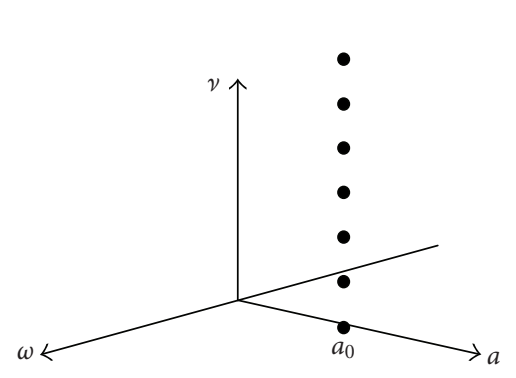

(d)

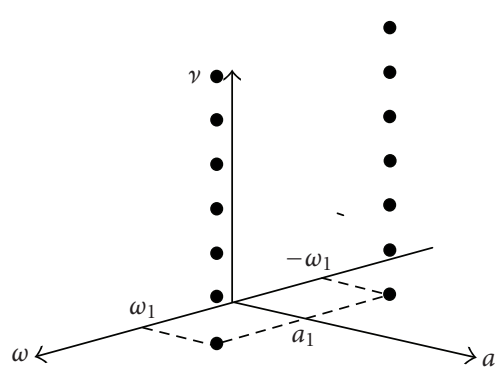

(e)

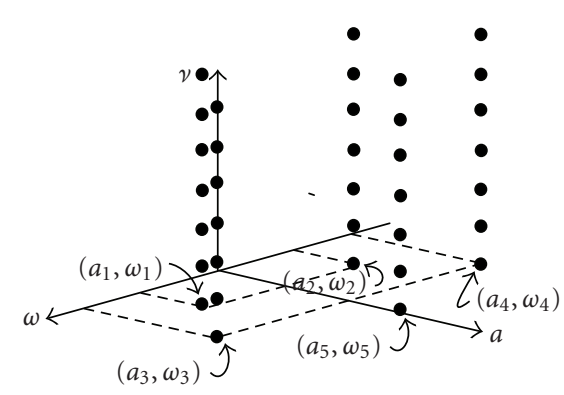

(f)

FIgUre 2: The labeling sets for the CUT, UT, and their sampled versions. (a) CUT : $M=\left\{(v, a, \omega) \in \mathbb{R}^{3}: v \geq 0, a>0\right\}$. (b) UT: $M_{d}=$ $\left\{(n, a, \omega) \in \mathbb{Z} \times \mathbb{R}^{2}: n \geq 0, a>0\right\}$. (c) Laplace : $M_{L}=\left\{(0, a, \omega) \in 0 \times \mathbb{R}^{2}: a \geq \varepsilon>0\right\}$. (d) Laguerre $: M_{a_{0}}=\left\{\left(n, a_{0}, 0\right) \in \mathbb{Z} \times\left(a_{0}, 0\right): n \geq 0\right\}$. (e) Kautz: $M_{2}=\left\{\left(n, a_{i}, \omega_{i}\right): n \in \mathbb{Z}, n \geq 0, i=1,2\right\}$. (f) Hambo: $M_{2}=\left\{\left(n, a_{i}, \omega_{i}\right): n \in \mathbb{Z}, n \geq 0, i=1, \ldots, N\right\}$.

as a labeling set (see Figure 2(a)) and define the measure

$$
\mu(A)=\iiint_{(\nu, a, \omega) \in A} \frac{\nu e^{-v}}{a^{2}} d \nu d a d \omega
$$

for any set $A \subseteq M$. This measure enables integrating measurable functions $F: M \rightarrow \mathbb{C}$. With this, we can write

$$
\|g\|_{L^{2}(\mu)}^{2} \equiv \iiint_{(\nu, a, \omega) \in M} \frac{\nu e^{-\nu}}{a^{2}}|g(\nu, a, \omega)|^{2} d \nu d a d \omega .
$$

Let $L^{2}(\mu)$ be the set of all $g$ 's such that $\|g\|_{\mu}^{2}<\infty$. Then $L^{2}(\mu)$ is a Hilbert space with inner product given by

$$
\left\langle g_{1}, g_{2}\right\rangle_{L^{2}(\mu)}=\int_{0}^{\infty} \int_{0}^{\infty} \int_{-\infty}^{\infty} \frac{\nu e^{-\nu}}{a^{2}} \overline{g_{1}(\nu, a, \omega)} g_{2}(\nu, a, \omega) \omega d \nu d a d
$$

which is the result of polarizing the norm in (14) (see [1, Theorem 1.12]).

We claim now that the set $\left\{\varphi_{\nu, a, \omega}\right\}_{(v, a, \omega) \in M}$ as given in (9) is a generalized frame in the space $L^{2}([0, \infty))$. First we note that the CUT equation, (18), can be viewed as the synthesis side of the frame operation, $f \mapsto \tilde{f}$, as given in (1) (Definition 1). The second part follows directly from the following lemma.

Lemma 1. For any $f \in L^{2}([0, \infty))$

$$
\|\tilde{f}\|_{L^{2}(\mu)}^{2}=2 \pi\|f\|_{2}^{2} .
$$

Proof. Using (11) and (14) we have

$$
\begin{aligned}
& \|\tilde{f}\|_{L^{2}(\mu)}^{2} \\
& =\int_{0}^{\infty} \int_{0}^{\infty} \int_{0}^{\infty} \frac{v e^{-v}}{a^{2}}|\tilde{f}(\nu, a, \omega)|^{2} d \omega d v d a \\
& =\int_{0}^{\infty} \int_{0}^{\infty} \int_{-\infty}^{\infty} \frac{2 a}{\Gamma(2 v+1)} \frac{v e^{-v}}{a^{2}}\left[\int_{0}^{\infty}(2 a t)^{v} e^{-a t} e^{j \omega t} \overline{f(t)} d t\right] \\
& \times\left[\int_{0}^{\infty}(2 a \tau)^{v} e^{-a \tau} e^{-j \omega \tau} f(\tau) d \tau\right] d \omega d \nu d a \\
& =\int_{0}^{\infty} \int_{0}^{\infty} \int_{0}^{\infty} \int_{0}^{\infty} \frac{2 a}{\Gamma(2 v+1)} \frac{\nu e^{-v}}{a^{2}} d v d a(2 a t)^{\nu}(2 a \tau)^{\nu} \\
& \times e^{-a(t+\tau)} \overline{f(t)} f(\tau) d t d \tau \\
& \times\left[\int_{-\infty}^{\infty} e^{j \omega(t-\tau)} d \omega\right] \\
& =2 \pi \int_{0}^{\infty}|f(t)|^{2} d t \int_{0}^{\infty} \frac{2 v e^{-v}}{\Gamma(2 v+1)} d v \\
& \times \int_{0}^{\infty}(2 a t)^{2 v-1} e^{-2 a t} 2 t d a \\
& =2 \pi \int_{0}^{\infty}|f(t)|^{2} d t \int_{0}^{\infty} \frac{e^{-v} 2 \nu \Gamma(2 v)}{\Gamma(2 v+1)} d \nu \\
& =2 \pi \int_{0}^{\infty}|f(t)|^{2} d t \int_{0}^{\infty} e^{-v} d v \\
& =2 \pi\|f\|_{2}^{2} \text {. }
\end{aligned}
$$

Clearly, (2) is satisfied with $A=B=2 \pi$ (which makes the frame a tight frame). Next we prove the following. 
Lemma 2. For any $f \in L^{2}([0, \infty))$

$$
f(t)=\frac{1}{2 \pi} \int_{-\infty}^{\infty} \int_{0}^{\infty} \int_{0}^{\infty} \frac{\nu e^{-\nu}}{a^{2}} \tilde{f}(\nu, a, \omega) \varphi_{\nu, a, \omega}(t) d \nu d a d \omega .
$$

Proof. Follows directly from Lemma 1 and [8].

Lemma 2 is in fact the synthesis side of the frame operation, $\tilde{f} \mapsto f$ and can also be viewed as the inverse CUT (ICUT).

We have defined a set of functions and showed that it is a (generalized) frame in $L^{2}([0, \infty))$. This frame is labeled by the continuous set $M$. It is thus, hardly surprising to find out that we can generate various subsets of this frame which are still (generalized) frames in $L^{2}([0, \infty))$. In fact, as we will show shortly, some of these subsets/frames result in wellknown transforms. Basically, these subsets will be chosen by a variety of sampling patterns in the labeling (transform) domain quite similar to the way one gets DWT from CWT. A similar idea can be found in, for example, [10].

\subsection{Generalized (sub) frames related to the CUT}

\subsubsection{The unified transform}

Let us consider the same functions with a labeling set $M_{d}=$ $\{(n, a, \omega): 0 \leq n \in \mathbb{Z}, a>0, \omega \in \mathbb{R}\} \subset M$ (see Figure 2(b)). We define the measure on this set by

$$
\mu_{d}(A)=\sum \iint_{(n, a, \omega) \in A} \frac{n e^{-n}}{a^{2}} d a d \omega
$$

resulting in modified definitions of the norm and inner product in the "transform" domain

$$
\begin{array}{r}
\|g(n, a, \omega)\|_{L^{2}\left(\mu_{d}\right)}=\sum_{n=0}^{\infty} \int_{-\infty}^{\infty} \int_{0}^{\infty} \frac{n e^{-n}}{a^{2}}|g(n, a, \omega)|^{2} d a d \omega, \\
\left\langle g_{1}, g_{2}\right\rangle_{L^{2}\left(\mu_{d}\right)}=\sum_{n=0}^{\infty} \int_{-\infty}^{\infty} \int_{0}^{\infty} \frac{n e^{-n}}{a^{2}} \overline{g_{1}(n, a, \omega)} g_{2}(n, a, \omega) d a d \omega .
\end{array}
$$

The corresponding analysis equation, referred to as the unified transform (UT), is then given by

$$
\begin{aligned}
\tilde{f}(n, a, \omega) & =\left\langle\varphi_{n, a, \omega}, f\right\rangle \\
& =\sqrt{\frac{2 a}{\Gamma(2 n+1)}} \int_{0}^{\infty}(2 a t)^{n} e^{-a t} e^{-j \omega t} f(t) d t
\end{aligned}
$$

and the synthesis, or the inverse UT (IUT), by

$$
\begin{aligned}
f(t) & =\int_{M} d \mu_{d}\left\langle\varphi_{n, a, \omega}, f\right\rangle \varphi_{n, a, \omega} \\
& =\frac{1-e^{-1}}{2 \pi} \sum_{n=0}^{\infty} \int_{-\infty}^{\infty} \int_{0}^{\infty} \frac{n e^{-n}}{a^{2}} \tilde{f}(n, a, \omega) \varphi_{n, a, \omega}(t) d a d \omega .
\end{aligned}
$$

Note that the UT results from sampling the CUT in the $v$ directions. Namely, $\tilde{f}(n, a, \omega)=\left.\tilde{f}(\nu, a, \omega)\right|_{\nu=n}$. From (20)-(22) it can be shown (quite similarly to the proof in Lemma 1) that the set $\left\{\varphi_{n, a, \omega}\right\}_{(n, a, \omega) \in M_{d}}$ is again a generalized, tight frame with frame bounds $A=B=2 \pi /\left(1-e^{-1}\right)$.

The main thrust of our discussion is the UT but, before discussing its properties we further "sample" the CUT (or, equivalently, choose various subsets of $\left\{\varphi_{v, a, \omega}\right\}$ ) and show that a number of well-known transforms result from this process.

\subsubsection{Laplace transform}

Let us consider now the same functions given by (9) with the restriction $v=0$, resulting in the labeling set $M_{L}=$ $\{(0, a, \omega): a>0, \omega \in \mathbb{R}\} \subset M$ (see Figure 2(c)). Then we readily note that

$$
\frac{1}{\sqrt{2 a}} \tilde{f}(0, a, \omega)=\frac{1}{\sqrt{2 a}}\left\langle\varphi_{0, a, \omega}, f\right\rangle=\int_{0}^{\infty} f(t) e^{-(a+j \omega) t} d t
$$

which is the definition of the (one-sided) Laplace transform (where $s=a+j \omega$ is the Laplace variable and since we assumed $f \in L^{2}([0, \infty)), a>0$ guarantees that we are always in the region of convergence). As we well know the inverse Laplace transform is (using our notation) $\left(1 /\left(2 \pi \sqrt{2 a_{0}}\right)\right) \int_{-\infty}^{\infty} \tilde{f}\left(0, a_{0}, \omega\right) e^{a_{0} t} e^{j \omega t} d \omega$, which means that it uses only $\tilde{f}\left(0, a_{0}, \omega\right)$ and reconstructs with the functions $\left(e^{a_{0} t} e^{j \omega t} /\left(2 \pi \sqrt{2 a_{0}}\right)\right)$ which are not in $L^{2}([0, \infty))$. It is thus, hardly surprising that the set of functions labeled by $M_{L}$ is not a frame. In fact, taking $\mu_{L}(A)=\int_{(a, \omega) \in A}\left(d a d \omega / a^{2}\right)$ it can be shown that $\|\tilde{f}(0, a, \omega)\|$ does not have an upper bound. It is however interesting to note that while it is not a frame it does have a reciprocal (dual) set of functions in $L^{2}([0, \infty))$. This is presented in the following lemma.

Lemma 3. The set of functions $\left\{(1 / 2 \pi \sqrt{2}) \varphi_{1, a, \omega}\right\}$ is reciprocal (biorthogonal) to $\left\{\varphi_{0, a, \omega}\right\}$.

Proof. With $\varphi_{0, a, \omega}^{*}$ denoting the adjoint of $\varphi_{0, a, \omega}$, by (9) we have

$$
\begin{aligned}
{\left[\int_{M_{L}}\right.} & \left.\frac{1}{2 \pi \sqrt{2}} \varphi_{1, a, \omega} \varphi_{0, a, \omega}^{*} d \mu_{L}\right] f(t) \\
= & \frac{1}{2 \pi \sqrt{2}} \int_{0}^{\infty} \int_{-\infty}^{\infty} \frac{d a d \omega}{a^{2}}\left[\sqrt{a}(2 a t) e^{-a t} e^{j \omega t}\right] \\
& \times\left[\sqrt{2 a} \int_{0}^{\infty} e^{-a \tau} e^{-j \omega \tau} f(\tau) d \tau\right] \\
= & \int_{0}^{\infty} d \tau f(\tau) \int_{0}^{\infty} 2 t e^{-a(t+\tau)} d a\left[\frac{1}{2 \pi} \int_{-\infty}^{\infty} e^{j \omega(t-\tau)} d \omega\right] \\
= & \int_{0}^{\infty} d \tau f(\tau) \int_{0}^{\infty} 2 t e^{-a(t+\tau)} \delta(\tau-t) d a \\
= & f(t) \int_{0}^{\infty} 2 t e^{-2 a t} d a \\
= & f(t) .
\end{aligned}
$$


This leads to the following reconstruction formula of $f(t)$ from $\tilde{f}(0, a, \omega)$ :

$$
f(t)=\frac{1}{2 \pi \sqrt{2}} \int_{0}^{\infty} \int_{-\infty}^{\infty} \frac{d a d \omega}{a^{2}} \varphi_{1, a, \omega}(t) \tilde{f}(0, a, \omega)
$$

which, in light of the observation (23) can be viewed as an inverse Laplace transform for functions in $L^{2}([0, \infty))$.

\subsubsection{Fourier transform}

Clearly, from (23), we have that $\left.((1 / \sqrt{2 a}) \tilde{f}(0, a, \omega))\right|_{a=0}$ is the Fourier transform of $f$. It is well known that for the resulting Fourier integral to exist, $f$ has to satisfy the condition $\int_{0}^{\infty}|f(t)|<\infty$. Furthermore, we can then readily see (since for $n \in \mathbb{N}, \Gamma(n+1)=n$ !) that

$$
\begin{aligned}
\sum_{n=0}^{\infty} \frac{\sqrt{\Gamma(2 n+1)}}{2^{n} \Gamma(n+1)} \frac{1}{\sqrt{2 a}} \tilde{f}(n, a, \omega) \\
\quad=\sum_{n=0}^{\infty} \frac{2^{-n}}{n !} \int_{0}^{\infty}(2 a t)^{n} e^{-a t} e^{-j \omega t} f(t) d t \\
=\int_{0}^{\infty} e^{-a t} e^{-j \omega t} f(t) \sum_{n=0}^{\infty} \frac{(a t)^{n}}{n !} d t \\
=\int_{0}^{\infty} e^{-a t} e^{-j \omega t} f(t) e^{a t} \\
=\int_{0}^{\infty} e^{-j \omega t} f(t) d t .
\end{aligned}
$$

In our derivation above we have exchanged the order of integration and summation. This is justified by using Fubini's theorem (see, e.g., [11]) and the fact that $\int_{0}^{\infty}|f(t)|<\infty$.

\subsubsection{Laguerre functions}

Let us now fix both the $a$ and $\omega$ variables to $a=a_{0}>0$ and $\omega=0$, respectively, and consider the labeling set $M_{a_{0}}=$ $\left\{\left(n, a_{0}, 0\right), 0 \leq n \in \mathbb{Z}\right\} \subset M$. Then the resulting set of functions, $\left\{\varphi_{k, a_{0}, 0}(t), k \in \mathbb{N}_{0}\right\}$, when taken through the GramSchmidt orthogonalization procedure (see (28) below), gives the well-known Laguerre orthonormal basis $\left\{\mathcal{L}_{r}(t), r \in \mathbb{N}_{0}\right\}$ in $L^{2}([0, \infty))$.

The Laguerre functions are characterized by a fixed pole $a_{0}$ and have the form

$$
\mathcal{L}_{r}(t)=\sqrt{2 a_{0}} \sum_{n=0}^{r}(-1)^{n}\left(\begin{array}{l}
r \\
n
\end{array}\right) \frac{\left(2 a_{0} t\right)^{n}}{n !} e^{-a_{0} t}, \quad r=0,1,2, \ldots,
$$

and it can be shown that $\left\langle\mathcal{L}_{r}, \mathcal{L}_{m}\right\rangle=\delta_{(r-m)}$ (Kronecker delta).

These functions can be written as a finite linear combination of the frame functions (9) and vice versa as stated in the following lemma.
Lemma 4. Let $\mathcal{L}_{r}(t)$ be the Laguerre functions (given in (27)). Then

$$
\begin{gathered}
\mathcal{L}_{r}(t)=\sum_{k=0}^{r} \alpha(r, k) \varphi_{k, a_{0}, 0}(t), \\
\varphi_{r, a_{0}, 0}(t)=\sum_{k=0}^{r} \beta(r, k) \mathcal{L}_{k}(t), \\
\sum_{k=n}^{r} \alpha(k, n) \beta(r, k)=\delta_{(n-r)},
\end{gathered}
$$

where the coefficients $\alpha(r, k), \beta(r, k), 0 \leq r, k \in \mathbb{Z}$ are defined by

$$
\begin{aligned}
& \alpha(r, k)= \begin{cases}(-1)^{k}\left(\begin{array}{l}
r \\
k
\end{array}\right) \frac{\sqrt{\Gamma(2 k+1)}}{k !} & \text { for } k \leq r, \\
0 & \text { otherwise, }\end{cases} \\
& \beta(r, k)= \begin{cases}(-1)^{k}\left(\begin{array}{l}
r \\
k
\end{array}\right) \frac{r !}{\sqrt{\Gamma(2 r+1)}} & \text { for } k \leq r, \\
0 & \text { otherwise. }\end{cases}
\end{aligned}
$$

Proof. Equation (28) follows immediately from (27) and (30). Using (30) we get

$$
\begin{aligned}
\sum_{k=n}^{r} \alpha(k, n) \beta(r, k) \\
=\sum_{k=n}^{r}(-1)^{k-n}\left(\begin{array}{l}
k \\
n
\end{array}\right)\left(\begin{array}{l}
r \\
k
\end{array}\right) \frac{r ! \sqrt{\Gamma(2 n+1)}}{\sqrt{\Gamma(2 r+1)} n !} \\
=\frac{(r !)^{2} \sqrt{\Gamma(2 n+1)}}{(n !)^{2} \sqrt{\Gamma(2 r+1)}} \sum_{k=n}^{r}(-1)^{k-n} \frac{1}{(k-n) !(r-k) !} \\
=\frac{(r !)^{2} \sqrt{\Gamma(2 n+1)}}{(r-n) !(n !)^{2} \sqrt{\Gamma(2 r+1)}} \sum_{\tilde{k}=0}^{r-n}(-1)^{\tilde{k}} \frac{(r-n) !}{\tilde{k} !(r-n-\tilde{k}) !} \\
=\frac{(r !)^{2} \sqrt{\Gamma(2 n+1)}}{(r-n) !(n !)^{2} \sqrt{\Gamma(2 r+1)}}(1-1)^{r-n} \\
=\delta_{(n-r)}
\end{aligned}
$$

which completes the proof of (29). Then, using this and (28) we readily get

$$
\begin{aligned}
\sum_{k=0}^{r} \beta(r, k) \mathcal{L}_{k}(t) & =\sum_{k=0}^{r} \beta(r, k) \sum_{m=0}^{k} \alpha(k, m) \varphi_{m, a_{0}, 0} \\
& =\sum_{m=0}^{r} \varphi_{m, a_{0}, 0} \sum_{k=0}^{r} \alpha(k, m) \beta(r, k) \\
& =\sum_{m=0}^{r} \varphi_{m, a_{0}, 0} \sum_{k=m}^{r} \alpha(k, m) \beta(r, k) \\
& =\sum_{m=0}^{r} \varphi_{m, a_{0}, 0} \delta_{(r-m)} \\
& =\varphi_{r, a_{0}, 0}
\end{aligned}
$$

which completes the proof of the lemma. 
With these relations we can create the reciprocal (dual) set for $\left\{\varphi_{k, a_{0}, 0}(t), k \in \mathbb{N}_{0}\right\}$, as is stated next.

Lemma 5. Define the functions $\varphi^{k, a_{0}, 0}(t), 0 \leq k \in \mathbb{Z}$, by

$$
\begin{aligned}
\varphi^{k, a_{0}, 0}(t) & =\sum_{m=k}^{\infty} \alpha(m, k) \sum_{l=0}^{m} \alpha(m, l) \varphi_{l, a_{0}, 0}(t) \\
& =\sum_{m=k}^{\infty} \alpha(m, k) \mathcal{L}_{m}(t) .
\end{aligned}
$$

Then, $\left\{\varphi^{k, a_{0}, 0}\right\}_{k=0}^{\infty}$ and $\left\{\varphi_{k, a_{0}, 0}\right\}_{k=0}^{\infty}$ are biorthogonal in the sense that

$$
\left\langle\varphi^{k, a_{0}, 0}, \varphi_{n, a_{0}, 0}\right\rangle=\delta_{(k-n)}
$$

Proof. By substitution of (33) we get

$$
\left\langle\varphi^{k, a_{0}, 0}, \varphi_{n, a_{0}, 0}\right\rangle=\sum_{m=k}^{\infty} \alpha(m, k)\left\langle\mathcal{L}_{m}, \varphi_{n, a_{0}, 0}\right\rangle .
$$

Then

$$
\begin{aligned}
\left\langle\varphi^{k, a_{0}, 0}, \varphi_{n, a_{0}, 0}\right\rangle & =\sum_{m=k}^{\infty} \alpha(m, k)\left\langle\mathcal{L}_{m}, \sum_{l=0}^{n} \beta(n, l) \mathcal{L}_{l}\right\rangle \\
& =\sum_{m=k}^{\infty} \alpha(m, k) \sum_{l=0}^{n} \beta(n, l) \delta_{l-m} \\
& =\sum_{m=k}^{\infty} \alpha(m, k) \beta(n, m) \\
& =\sum_{m=k}^{n} \alpha(m, k) \beta(n, m) \\
& =\delta_{(k-n)}
\end{aligned}
$$

which completes the proof.

Lemma 5 and the observation that $\left\{\varphi^{k, a_{0}, 0}\right\}_{k=0}^{\infty}$ span $L^{2}([0, \infty))$, naturally lead to the reconstruction (synthesis or inverse transform)

$$
\begin{aligned}
f(t) & =\sum_{k=0}^{\infty}\left\langle\varphi_{k, a_{0}, 0}, f\right\rangle \varphi^{k, a_{0}, 0}(t) \\
& =\sum_{k=0}^{\infty} \tilde{f}\left(k, a_{0}, 0\right) \varphi^{k, a_{0}, 0}(t) .
\end{aligned}
$$

\subsubsection{Hambo transform and the Kautz result}

In this section we extend the label set which led to the Laguerre functions by considering a finite set of pairs $\left(a_{i}, \omega_{i}\right)$ and define $M_{2}=\left\{\left(n, a_{i}, \omega_{i}\right): 0 \leq n \in \mathbb{Z}, 1 \leq i \leq N\right\}$ where for every $\omega_{j} \neq 0$ we have $\left(n, a_{j}, \omega_{j}\right) \in M_{2} \Leftrightarrow\left(n, a_{j},-\omega_{j}\right) \in$ $M_{2}$. The corresponding set can be viewed as a union of $N$ sets $M_{a_{0}}$ of the previous subsection. By ordering the set so that the $k$ th function is such that $k=n N+i$ and then orthogonalizing one gets the Hambo basis which corresponds to the Hambo transform. The case with $N=2$ is known in the literature as the Kautz functions (see, e.g., [2]).
It is interesting to note that if the orthogonalization is carried out along each $i$ separately, one gets $N$ orthonormal bases. The union of these bases is known to be a (tight) frame with bound equal to $N$.

An alternative choice of subset of functions can be generated when we let $N \rightarrow \infty$ in the above $M_{2}$ but $n$ may be finite. This includes any general sampling pattern of the original labeling set $M$. Whether the resulting set of functions is indeed a (generalized) frame or not is very closely related to the result of Kautz and the condition derived by Szas (see, e.g., in [12]).

\section{PROPERTIES OF THE UNIFIED TRANSFORM}

As stated earlier, our main interest is in the unified transform (UT). We recall its definition

$$
\begin{aligned}
\tilde{f}(n, a, \omega) & =\left\langle\varphi_{n, a, \omega}, f\right\rangle \\
& =\sqrt{\frac{2 a}{\Gamma(2 n+1)}} \int_{0}^{\infty}(2 a t)^{n} e^{-a t} e^{-j \omega t} f(t) d t
\end{aligned}
$$

and the inverse transform is given by (22):

$$
f(t)=\frac{1-e^{-1}}{2 \pi} \sum_{n=0}^{\infty} \int_{0}^{\infty} \int_{0}^{\infty} d a d \omega \frac{n e^{-n}}{a^{2}} \tilde{f}(n, a, \omega) \varphi_{n, a, \omega}(t) .
$$

In the next lemma we summarize some of its properties.

Lemma 6. The unified transform has the following properties.

(1) Time derivative: let $f_{1}(t)=(d f(t) / d t)$, then

$$
\tilde{f}_{1}(0, a, \omega)=(a+j \omega) \tilde{f}(0, a, \omega)-\sqrt{2 a} f(0),
$$

and for $n \geq 1$,

$$
\tilde{f}_{1}(n, a, \omega)=(a+j \omega) \tilde{f}(n, a, \omega)-a \sqrt{\frac{2 n}{2 n-1}} \tilde{f}(n-1, a, \omega) .
$$

(2) Time shift: let $f_{1}(t)=f(t-T)$, then

$$
\begin{aligned}
& \tilde{f}_{1}(n, a, \omega) \\
& \quad=e^{-T(a+j \omega)} \sum_{m=0}^{n}\left(\begin{array}{l}
n \\
m
\end{array}\right)(2 a T)^{n-m} \sqrt{\frac{\Gamma(2 m+1)}{\Gamma(2 n+1)}} \tilde{f}(m, a, \omega) .
\end{aligned}
$$

(3) Convolution: let $y(t)=g(t) * u(t)=\int_{0}^{\infty} g(\sigma) u(t-$ $\sigma) d \sigma$, then

$$
\begin{aligned}
\tilde{y}(n, a, \omega)=\sum_{m=0}^{n}\left(\begin{array}{l}
n \\
m
\end{array}\right) \sqrt{\frac{\Gamma(2 m+1) \Gamma(2(n-m)+1)}{2 a \Gamma(2 n+1)}} \\
\times \tilde{u}(m, a, \omega) \tilde{g}(n-m, a, \omega) .
\end{aligned}
$$

Note that if a normalized version of the transform is defined as

$$
\hat{y}(n, a, \omega)=\frac{\sqrt{\Gamma(2 n+1)}}{n !} \tilde{y}(n, a, \omega)
$$


(43) can be rewritten as

$$
\hat{y}(n, a, \omega)=\frac{1}{\sqrt{2 a}} \sum_{m=0}^{n} \hat{g}(n-m, a, \omega) \hat{u}(m, a, \omega)
$$

which is clearly a linear convolution along the n-axis.

(4) Derivative with respect to $\omega$ :

$$
\frac{\partial \tilde{f}(n, a, \omega)}{\partial \omega}=\frac{1}{\sqrt{2} a j} \sqrt{(2 n+1)(n+1)} \tilde{f}(n+1, a, \omega) .
$$

(5) Derivative with respect to a:

$$
\begin{aligned}
\frac{\partial \tilde{f}(n, a, \omega)}{\partial a}= & \frac{1}{a}\left(n+\frac{1}{2}\right) \tilde{f}(n, a, \omega) \\
& -\frac{1}{\sqrt{2} a} \sqrt{(2 n+1)(n+1)} \tilde{f}(n+1, a, \omega) .
\end{aligned}
$$

\subsection{LTI system representation in the transform domain}

To simplify our discussion we restrict ourselves to singleinput single-output (SISO) LTI systems. We next investigate what form an LTI system takes on in the transform domain. As is well known, there are a number of equivalent LTI system representations (convolution, differential equations, state space, etc.). We could start with any of them and show the equivalence of the results in the transform domain. However, we feel it will suffice to investigate one of them and we chose the state space representation.

Consider the SISO LTI system given by

$$
\begin{gathered}
\frac{d}{d t} x(t)=A x(t)+B u(t), \quad x(0)=x_{0}, \\
y(t)=C x(t)+D u(t),
\end{gathered}
$$

where $x(t) \in \mathbb{R}^{L}$. Applying the transform $((21))$ on both sides and using property (1) of Lemma 6 we can show that the transforms of the input and the output satisfy the following difference equations for $n \geq 0$ :

$$
\begin{gathered}
(a+j \omega) \tilde{x}(0, a, \omega)-\sqrt{2 a} x(0)=A \tilde{x}(0, a, \omega)+B \tilde{u}(0, a, \omega) \\
(a+j \omega) \tilde{x}(n+1, a, \omega)-a \sqrt{\frac{2 n+2}{2 n+1}} \tilde{x}(n, a, \omega) \\
=A \tilde{x}(n+1, a, \omega)+B \tilde{u}(n+1, a, \omega) .
\end{gathered}
$$

Substituting

$$
\begin{aligned}
& \tilde{X}(n, a, \omega)=\frac{\tilde{x}(n, a, \omega)-((a+j \omega) I-A)^{-1} B \tilde{u}(n, a, \omega)}{d_{n}}, \\
& \tilde{U}(n, a, \omega)=\frac{\tilde{u}(n, a, \omega)}{d_{n}}, \\
& \tilde{Y}(n, a, \omega)=\frac{\tilde{y}(n, a, \omega)}{d_{n}},
\end{aligned}
$$

where

$$
d_{n}=\frac{2^{n} \Gamma(n+1)}{\sqrt{\Gamma(2 n+1)}}
$$

we get the discrete time state space form

$$
\begin{gathered}
\tilde{X}(n+1, a, \omega)=\tilde{A}(a, \omega) \tilde{X}(n, a, \omega)+\widetilde{B}(a, \omega) \tilde{U}(n, a, \omega), \\
\tilde{Y}(n, a, \omega)=\widetilde{C}(a, \omega) \tilde{X}(n, a, \omega)+\widetilde{D}(a, \omega) \tilde{U}(n, a, \omega),
\end{gathered}
$$

where

$$
\begin{aligned}
\widetilde{A}(a, \omega) & =a((a+j \omega) I-A)^{-1}, \\
\widetilde{B}(a, \omega) & =a((a+j \omega) I-A)^{-2} B, \\
\widetilde{C}(a, \omega) & =C \\
\widetilde{D}(a, \omega) & =D+C((a+j \omega) I-A)^{-1} B,
\end{aligned}
$$

and initial conditions

$$
\begin{aligned}
\tilde{X}(0, a, \omega) & =\tilde{x}(0, a, \omega)-((a+j \omega) I-A)^{-1} B \tilde{u}(0, a, \omega) \\
& =\sqrt{2 a}((a+j \omega) I-A)^{-1} x_{0} .
\end{aligned}
$$

Remark 1. When we restrict the labeling set to $M_{2}$ (see Section 2.2.5) the results above are in agreement with the results in [13] regarding the Hambo transform.

\section{COMMENTS REGARDING THE USE OF UT FOR SYSTEM IDENTIFICATION}

We wish to stress here, again, the important potential for system identification we see in representing a function in $L^{2}([0, \infty))$, using the (generalized) frame $\left\{\varphi_{n, a, \omega}\right\}_{(n, a, \omega) \in M_{d}}$. It stems from the observation that the impulse response of every finite-dimensional stable LTI system has a finite representation in this set. More specifically, as is well known, the impulse response of every $N$-dimensional stable LTI system can be written as

$$
h(t)=\sum_{i=1}^{I} \sum_{n=0}^{N_{i}-1} c_{i, n} t^{n} e^{\left(-a_{i}+j \omega_{i}\right)}
$$

where $N=\sum_{i=1}^{I} N_{i}$ is the system dimension and $\left\{\left(-a_{i}+\right.\right.$ $\left.\left.j \omega_{i}\right)\right\}_{i=1}^{I}$ are the system distinct poles (each with respective repetition of $N_{i}$ ). Then, clearly

$$
h(t)=\sum_{i=1}^{I} \sum_{n=0}^{N_{i}-1} \tilde{c}_{i, n} \varphi_{n, a_{i}, \omega_{i}}(t) .
$$

The UT we defined is only one (out of infinitely many) possible representations of a given signal in this frame. It can be shown to be optimal in the least square sense. Namely, of all functions $g(n, a, \omega)$ which are representations of a given function $f$ in this frame, $\tilde{f}$ is the one with least energy (see for more detail [1]). However, in order to find the sparsest representation, a different optimization criterion will be needed. Specifically, for a function such as $h(t)$ above, we 
know that there exists a finite representation (readily observed to be unique!) and we would like to have an optimization criterion which will render this particular representation as its optimum. This particular problem is of much interest and has generated, in finite-dimensional spaces, many published results (see, e.g., $[14,15]$ ).

We are currently investigating the possibilities of using different optimization criteria to generate different representations in the transform domain. Specifically, we are currently testing the possibilities of using $L^{1}$ norms in order to generate the sparsest representations.

A very relevant observation is summarized in the following lemma.

Lemma 7. Consider the function

$$
g(t)=\varphi_{l, \sigma, \Omega}(t)\left(=\sqrt{\frac{2 \sigma}{\Gamma(2 l+1)}}(2 \sigma t)^{l} e^{(-\sigma+j \Omega) t}\right)
$$

Then

$$
\forall(n, a, \omega) \neq(l, \sigma, \Omega) \quad|\tilde{g}(l, \sigma, \Omega)|^{2}>|\tilde{g}(n, a, \omega)|^{2} .
$$

Proof. As $\tilde{g}(n, a, \omega)=\left\langle\varphi_{n, a, \omega}, g\right\rangle$ and $g(t)=\varphi_{l, \sigma, \Omega}(t)$, by Cauchy Schwarz we get

$$
\begin{aligned}
|\tilde{g}(n, a, \omega)| & \leq\left\|\varphi_{n, a, \omega}\right\|\left\|\varphi_{l, \sigma, \Omega}\right\| \\
& \leq 1
\end{aligned}
$$

where equality holds if and only if $\varphi_{n, a, \omega}(t)=\varphi_{l, \sigma, \Omega}(t)$, namely $(n, a, \omega)=(l, \sigma, \Omega)$. Then (58) follows since $\tilde{g}(l, \sigma, \Omega)=$ $\left\langle\varphi_{l, \sigma, \Omega}, g\right\rangle=\left\langle\varphi_{l, \sigma, \Omega}, \varphi_{l, \sigma, \Omega}\right\rangle=1$.

Lemma 7 means that if the system contains a single pole at $(-\sigma+j \Omega)$ with multiplicity $(l+1)$, finding the maximum of its UT will render both the pole location and its multiplicity.

\section{ACKNOWLEDGMENT}

We wish to thank the anonymous reviewer for his/her very thorough review which enabled us to make our results more precise and better presented.

\section{REFERENCES}

[1] G. Kaiser, A Friendly Guide to Wavelets, Birkhäuser, Boston, Mass, USA, 1994.

[2] P. S. C. Heuberger, T. J. de Hoog, P. M. J. Van den Hof, and B. Wahlberg, "Orthonormal basis functions in time and frequency domain: Hambo transform theory," SIAM Journal on Control and Optimization, vol. 42, no. 4, pp. 1347-1373, 2003.

[3] A. V. Oppenheim, A. S. Willsky, and I. T. Young, Signals and Systems, Prentice-Hall, Englewood Cliffs, NJ, USA, 1983.

[4] M. Vetterli and J. Kovacevic, Wavelets and Subband Coding, Prentice-Hall, Englewood Cliffs, NJ, USA, 1995.

[5] S. Mallat, A Wavelet Tour of Signal Processing, Academic Press, San Diego, Calif, USA, 2nd edition, 1999.

[6] I. Daubechies, Ten Lectures on Wavelets, CBMS-NSF Regional Conference Series in Applied Mathematics, SIAM, Philadelphia, Pa, USA, 1992.
[7] C. K. Chui, An Introduction to Wavelets, Wavelet Analysis and Its Applications, Academic Press, Boston, Mass, USA, 1992.

[8] O. Christensen, An Introduction to Frames and Riesz Bases, Birkhäuser, Boston, Mass, USA, 2003.

[9] S. T. Ali, J. P. Antoine, and J. P. Gazeau, "Continuous frames in Hilbert space," Annals of Physics, vol. 222, no. 1, pp. 1-37, 1993.

[10] E. Viscito and J. P. Allebach, "The analysis and design of multidimensional FIR perfect reconstruction filter banks for arbitrary sampling lattices," IEEE Transactions on Circuits And Systems-Part I : Fundamental Theory and Applications, vol. 38, no. 1, pp. 29-41, 1991.

[11] T. M. Apostol, Mathematical Analysis, Addison-Wesley, Sydney, Australia, 2nd edition, 1974.

[12] R. E. A. C. Paley and N. Wiener, Fourier Transforms in the Complex Domain, American Mathematical Society, New York, NY, USA, 1934

[13] T. J. de Hoog, Rational orthonormal bases and related transforms in linear system modeling, Ph.D. thesis, Delft University of Technology, Delft, The Netherlands, 2001.

[14] D. L. Donoho and P. B. Stark, "Uncertainty principles and signal recovery," SIAM Journal on Applied Mathematics, vol. 49, no. 3, pp. 906-931, 1989.

[15] D. L. Donoho and M. Elad, "Optimally sparse representation in general (nonorthogonal) dictionaries via $\ell^{1}$ minimization," Proceedings of the National Academy of Sciences of the United States of America, vol. 100, no. 5, pp. 2197-2202, 2003.

Arie Feuer has been with the Department of Electrical Engineering at the TechnionIsrael Institute of Technology since 1983, where he is currently a Professor and Head of the Control and Robotics Lab. He received his B.S. and M.S. degrees from the Technion in 1967 and 1973, respectively, and his Ph.D. degree from Yale University in 1978. From 1967 to 1970 he was in industry working on automation design and between

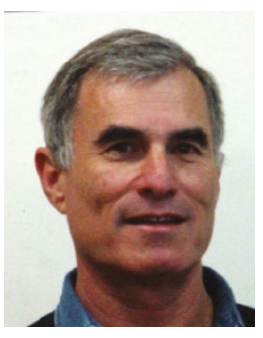
1978 and 1983 with Bell Labs, Holmdel. Between the years 1994 and 2002, he served as the President of the Israel Association of Automatic Control and is currently a Member of the IFAC Council. In the last 15 years he has been regularly visiting the Electrical Engineering and Computer Science Department at the University of Newcastle. His current research interests include the following. (1) Resolution enhancement of digital images and videos. (2) Sampling and combined representations of signals and images. (3) Adaptive systems in signal processing and control.

Paul M. J. Van den Hof was born in 1957 in Maastricht, The Netherlands. He received the M.S. and Ph.D. degrees both from the Department of Electrical Engineering, Eindhoven University of Technology, The Netherlands, in 1982 and 1989, respectively. Since 1999, he has been a Full Professor in the Signals, Systems, and Control Group of the Department of Applied Physics at Delft University of Technology, and since

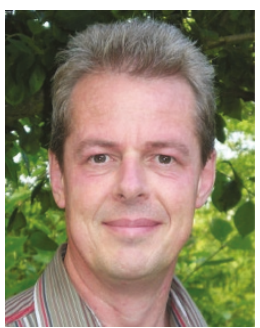
2003, he has been Codirector of the Delft Center for Systems and Control, with appointments in the Faculty of Mechanical Engineering and the Faculty of Applied Sciences. Since 2005, he has been Acting Scientific Director of the Dutch Institute of Systems and Control (DISC). Paul Van den Hof's research interests are in 
issues concerning system identification, parametrization, signal processing, and (robust) control design, with applications in mechanical servo systems, physical measurement systems, and industrial process control systems. He is a Member of the IFAC Council (1999-2005), Member of the Board of Governors of IEEE's Control System Society (2003-2005), and Automatica Editor for Rapid Publications.

Peter S. C. Heuberger was born in Maastricht, The Netherlands, in 1957. He obtained the M.S. degree in mathematics from the University of Groningen in 1983, and the Ph.D. degree from the Mechanical Engineering Department of the Delft University of Technology in 1991. He is a staff member of the Netherlands Environmental Assessment Agency (MNP) and also holds a parttime research position at the Delft Center

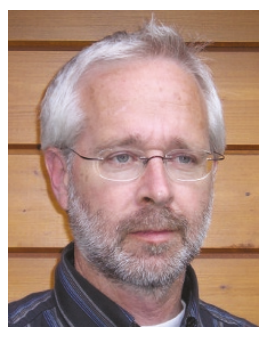
for Systems and Control (DCSC). His research interests are in issues of system identification and approximation, optimization, uncertainty and sensitivity analysis, model reduction, and in the theory and application of orthogonal basis functions. 